\title{
FACTORES DE INFORMACIÓN MEDIOAMBIENTAL DE LAS EMPRESAS MINERAS PERUANAS
}

\section{ENVIRONMENTAL REPORTING FACTORS OF PERUVIAN MINING COMPANIES}

\author{
Julio Hernández Pajares ${ }^{1}$, Cecilia Venegas Morales ${ }^{2}$, Luis Alvarado Pintado ${ }^{3}$
}

\section{RESUMEN}

Con el fin de satisfacer las expectativas de sus grupos de interés, las empresas mineras peruanas vienen comprometiendo sus esfuerzos sobre su desempeño de sostenibilidad y su reporte con el fin de mejorar su impacto social y ambiental así como su transparencia. El objetivo de la presente investigación es analizar el nivel de cumplimiento de la información medioambiental publicada por estas empresas en sus memorias anuales y de sostenibilidad, y determinar si factores como rentabilidad, tamaño y naturaleza de los accionistas influyen en el cumplimiento de dicha información de acuerdo a los estándares de la Global Reporting Initiative (GRI). Los resultados muestran que el tamaño de las empresas y la naturaleza de sus accionistas resultan factores determinantes de la información medioambiental.

Palabras claves: Accountability, Información Medioambiental, Minería Peruana, Responsabilidad Social Corporativa.

Recepción: 3/01/2017. Aprobación: 17/08//2017.

\begin{abstract}
Considering the expectations of its stakeholders, the Peruvian mining companies are committing their efforts on their sustainability performance and reporting in order to improve their social and environmental impact as well as their transparency. The aim of this research is to analyze the level of compliance of the environmental disclosure by these companies in their annual and sustainability reports, and whether factors such as profitability, size and shareholder nature influence the level of such information according to the criteria of the Global Reporting Initiative (GRI). The results show that there is significative influence between company size and nature of shareholders and level of environmental reporting.
\end{abstract}

Keywords: Accountability, Corporate Social Responsibility, Environmental Reporting, Peruvian Mining.

1 Área Académica de Contabilidad y Auditoría de la Universidad de Piura, Perú, julio.hernandez@udep.pe

2 Área Académica de Contabilidad y Auditoría de la Universidad de Piura, Perú, cecilia.venegas@udep.pe

3 Programa Académico de Administración de Empresas de la Universidad de Piura, Perú. luis.alvarado@udep.pe 


\section{INTRODUCCIÓN}

La Responsabilidad Social Corporativa (RSC) ha sido el marco, los últimos años, para que las empresas mineras incorporen en sus objetivos y gestión compromisos referentes al desarrollo sostenible con el fin de satisfacer las necesidades de los grupos de interés más influyentes. En el Perú estas empresas llevan a cabo un importante desempeño de sostenibilidad que responde a las expectativas de sus grupos de interés que comprenden, principalmente, las comunidades campesinas, buscando que la explotación minera y uso de recursos naturales produzcan el menor impacto en el medioambiente a la vez que contribuyen a su desarrollo social.

La actividad minera, especialmente en el Perú, ha establecido relaciones con sus grupos de interés que permiten la ejecución de sus operaciones a la vez que contribuyan al desarrollo sostenible; es decir, no solo realizan un desempeño ambiental y social responsable sino que buscan satisfacer los derechos de las comunidades con quienes interactúa a la vez de realizar un cumplimiento de regulaciones ambientales (Pino et al., 2010; Slack, 2012; Jaskoski, 2014; Helwege, 2014).

Como objetivo de la presente investigación, se plantea analizar el nivel de cumplimiento de la información medioambiental publicada por las empresas mineras peruanas en sus memorias anuales y de sostenibilidad en los años 2009 y 2010 de acuerdo a los estándares de la Global Reporting Initiative (GRI) y determinar si factores como rentabilidad, tamaño y naturaleza de los accionistas influyen en dicho nivel de información.

\section{ANTECEDENTES}

La actividad minera en el Perú ha permitido un aporte determinante a las exportaciones en 23,030 millones de dólares, que representaron el 55\% de las exportaciones totales y constituyeron una contribución del 2,2\% al Producto Bruto Interno del año $2013^{4}$.

La investigación sobre RSC y sostenibilidad de empresas mineras ha estado orientada al estudio del desempeño e información de sostenibilidad debido a su relevancia en la economía y su impacto en el desarrollo sostenible, especialmente en sus actuaciones ambientales y sociales con las comunidades locales (Hilson y Basu, 2003; Jenkins, 2004; Jenkins y Yakovleva, 2006; Guenther et al., 2006; Fonseca et al., 2014; Gifford et al., 2010; Prno, 2013).

Los estudios de empresas mineras han resultado relevante por su impacto ambiental con las comunidades campesinas que se ven afectadas por las actividades de extracción, tratamientos de materiales y residuos. En algunos casos dicho impacto ha creado conflictos ambientales y sociales y ha impedido que las empresas obtuvieran una licencia social para su operación con aprobación (Muradian et al., 2003; Jenkins, 2004; Bebbington y Bury, 2009).

La información de desempeño de RSC y medioambiental es una práctica responsable de las empresas mineras, que corresponde a una rendición de cuentas o accountability con sus grupos de interés sobre la gestión de los recursos que le son proveídos (Gray et al., 1996).

Esta rendición de cuentas corporativa ha comprendido la publicación en memorias de sostenibilidad, aspectos sobre desempeño económico, ambiental y social, de acuerdo con los criterios de la Guía de la GRI (GRI, 2006) en la mayoría de casos.

Sin embargo algunos estudios consideran que la publicación de estas memorias de sostenibilidad, sobre la rendición de cuentas del uso de los recursos naturales e impacto ambiental, son una forma de legitimación de la actividad minera frente a las comunidades donde operan y a la sociedad desde un punto de vista positivo y no encontrándose una rendición de cuentas que comprenda el contexto y el desempeño de sostenibilidad de largo plazo y compromiso con los grupos de interés (Jenkins y Yakovleva, 2006; Guenther et al., 2006; Gifford y Kestler, 2008; Gifford et al., 2010; Fonseca et al., 2014).

Otras investigaciones sobre memorias de sostenibilidad encuentran relaciones entre el cumplimiento o nivel de información y otros factores. Es así que se encuentra relación entre la información medioambiental y el tamaño de las empresas (Deegan y Gordon, 1996; Patten, 2002; Pérez y Sánchez, 2009; Da Silva y Aibar, 2011); su rentabilidad (Roberts, 1992; Moneva y Llena, 1996; Archel y Lizarraga, 2001) y la naturaleza de sus accionistas (Patten, 1992; Roberts, 1992; Da Silva y Aibar, 2011).

4 Anuario Minero 2013. Minería, Ministerio de Energía y Minas. Acceso en http://www.minem.gob.pe/_publicacion.php?idSector=1\&idPublicaci on $=477$. Consultado el 30 de junio de 2014 . 
De acuerdo al marco teórico revisado, para establecer si el tamaño, rentabilidad y naturaleza de los accionistas son factores determinantes en el nivel de cumplimiento de información ambiental para las empresas mineras peruanas, se plantean las siguientes hipótesis:

H1: Existe diferencia significativa en el nivel de cumplimiento de información ambiental según la rentabilidad de las empresas.

H2: Existe diferencia significativa en el nivel de cumplimiento de información ambiental según el tamaño de las empresas.

H3: Existe diferencia significativa en el nivel de cumplimento de información ambiental según la naturaleza de los accionistas de las empresas.

\section{METODOLOGÍA}

Para esta investigación se ha aplicado la metodología de análisis de contenido, aplicada en estudios como los de Gray et al. (1995); Llena et al. (2007); Moneva et al. (2007); Sweeney y Coughlan (2008), de acuerdo con los criterios e indicadores señalados por la Guía de la GRI (2006) y el Suplemento del Sector de Minería (GRI, 2010). El análisis de contenido se ha realizado sobre los informes anuales y de sostenibilidad de 43 empresas mineras, con respecto a su información de sostenibilidad de los años 2009 y 2010 que han sido publicadas en la base de datos de la GRI ${ }^{5}$.

Para esto se elaboró una herramienta de análisis que comprende el estudio de información medioambiental, bajo los aspectos de estrategias ambientales, compromisos y certificaciones internacionales, sistema de gestión y los indicadores económicos, sociales y ambientales (gestión de materiales, residuos, emisiones, agua, energia y biodiversidad) resumidos en 76 variables que explican el comportamiento de las empresas mineras en las memorias de sostenibilidad basadas en las Guías de la GRI y nueve variables en las memorias anuales que incluyen aspectos sobre activos, pasivos contingentes, gastos ambientales y revelaciones en notas a los estados financieros.

El análisis de contenido permitió aplicar una escala cuyo objeto es detectar la presencia (valor 1) o ausencia (valor 0) de información relativa a cada variable o elemento sin considerar la calidad o extensión de la información divulgada (Llena et al., 2007; Pérez y Sánchez, 2009). Para calcular la fiabilidad o exactitud de los datos en cuanto a su estabilidad, repetición o precisión, se aplicó la medida del Alfa de Cronbach; el análisis dio como resultado un factor 0.957 , lo que indica una adecuada consistencia interna de la herramienta usada para recoger información.

\section{RESULTADOS}

Para una mejor observación de las variables de estudio sobre el desempeño ambiental, se aplicó un análisis factorial que determinó los componentes que explican el tipo de información de sostenibilidad.

La prueba de adecuación muestral de Kaiser-Meyer-Olkin arrojó un coeficiente de 0.807, y la prueba de esfericidad de Bartlett resultó en un p-valor de 0.000 , lo que señala que las variables están correlacionadas. El análisis factorial proporciona cinco componentes, que logran reproducir el $62.15 \%$ de la varianza acumulada explicada.

Haciendo un análisis de los componentes rotados, los factores resultantes comprenden, un primer componente (MR) que se relaciona con las variables de desempeño ambiental sobre uso de materiales, gestión de residuos; el segundo, (AE), considera las variables sobre las políticas y estrategias de sostenibilidad, información sobre el cumplimiento de estándares de actividades extractivas e información sobre valoración de las contingencias ambientales; el tercero, (EE), referido a las variables de desempeño sobre consumo de energía y control de emisiones; el cuarto componente (PA), contempla los indicadores sobre la gestión y prevención ambiental, proyectos de desarrollo de infraestructura social y ambiental y auditorías ambientales; finalmente el quinto componente, (AT), explica las variables de información sobre políticas de capacitación al personal en asuntos ambientales, información sobre uso del agua, impactos en la biodiversidad.

Para un mejor estudio de la relación del nivel de información y las variables de estudios (rentabilidad, tamaño y naturaleza de los ac-

5 Global Reporting Initiative, http:/ / database.globalreporting.org/search. Consultado el 15 de octubre de 2013. 
cionistas), se determinaron cuatro grupos de empresas, en la aplicación de un análisis cluster bajo el método Ward, considerando los componentes del análisis factorial.

Los grupos obtenidos del análisis según los componentes se aprecian en el Gráfico 1. El primer grupo de empresas (1. ambientales) con una mayor prevención ambiental y gestión con grupos de interés, es el de mayor número de empresas y destacan en su información sobre impactos ambientales, con un claro desempeño con las comunidades afectadas por su actividad como señalan Gifford y Kestler (2008).

Gráfico 1: Grupos y componentes del análisis factorial.

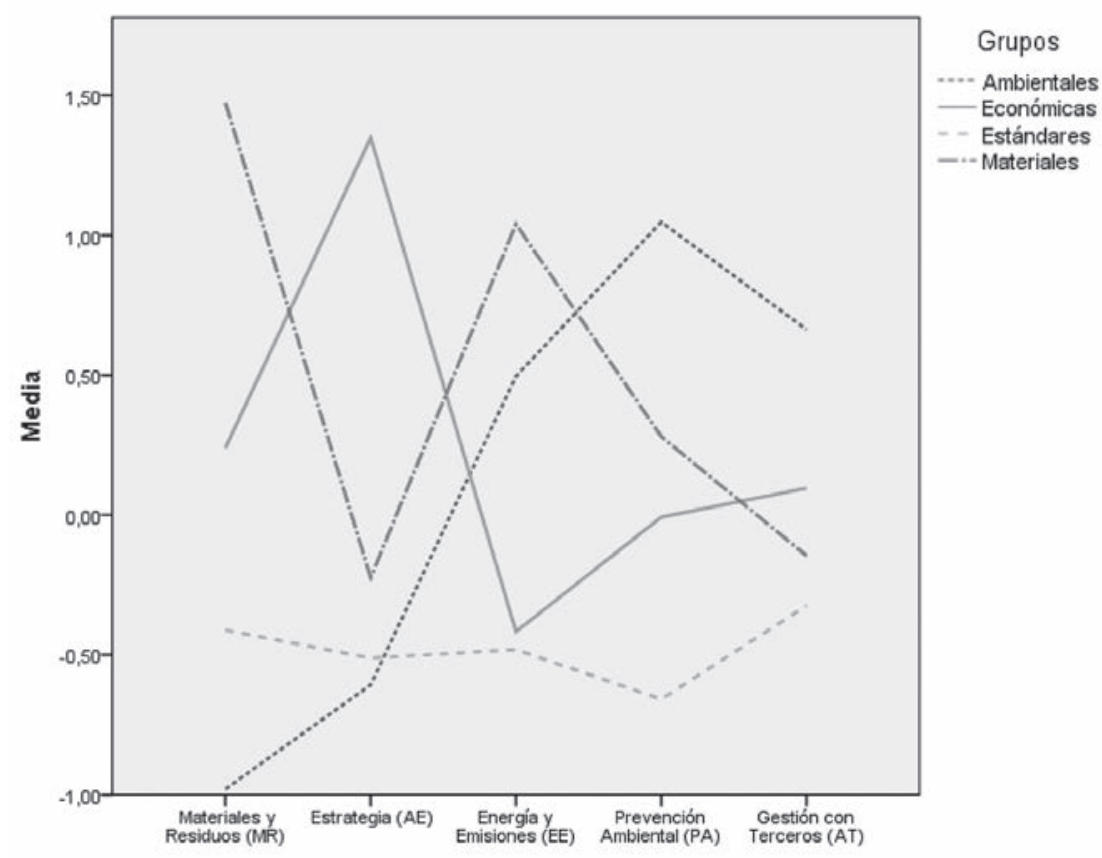

Fuente: Elaboración propia.

El segundo grupo (2. económicas) comprende las empresas que revelan información ambiental sobre desempeño económico y financiero y contingencias ambientales, además de sus estrategias de sostenibilidad. El tercer grupo (3. estándares) lo conforman aquellas que presentan un desempeño ambiental de cumplimiento mínimo de estándares de información. Finalmente, el cuarto grupo (4. materiales) comprende las empresas que divulgan mayor información sobre gestión ambiental de materiales, residuos, desechos y relaves y gestión de residuos. Estos resultados también señalan una falta de uniformidad de la información de sostenibilidad de las empresas mineras.

\section{Rentabilidad}

Para determinar si existe diferencia significativa se ha analizado el indicador de rentabilidad basado en el ROE de los años 2009 y 2010 para cada uno de los grupos como se observa en los Gráficos 2 y 3, cabe destacar que las empresas con mejor nivel de rentabilidad son aquellas que realizan una mayor gestión con grupos de interés (comunidades) y actividades de prevención y gestión ambiental. 
Gráfico 2: Rentabilidad 2009 por grupos.

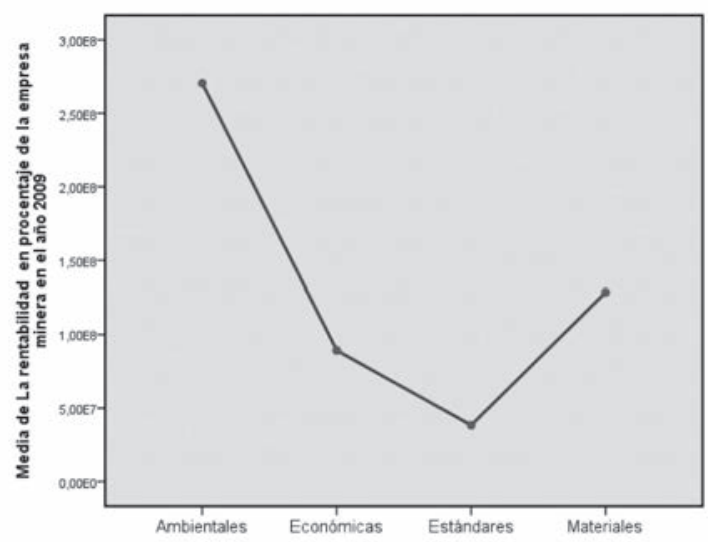

Fuente: Elaboración propia.

Para determinar si se presenta una diferencia significativa en los indicadores de rentabilidad se aplica una prueba ANOVA (Kruskal-Wa1lis), encontrando que no se presenta diferencia significativa de la variable rentabilidad para cada uno de los grupos, en la Tabla I se muestran p-valores mayores al nivel de significancia
Grafico 3: Rentabilidad 2010 por grupos.

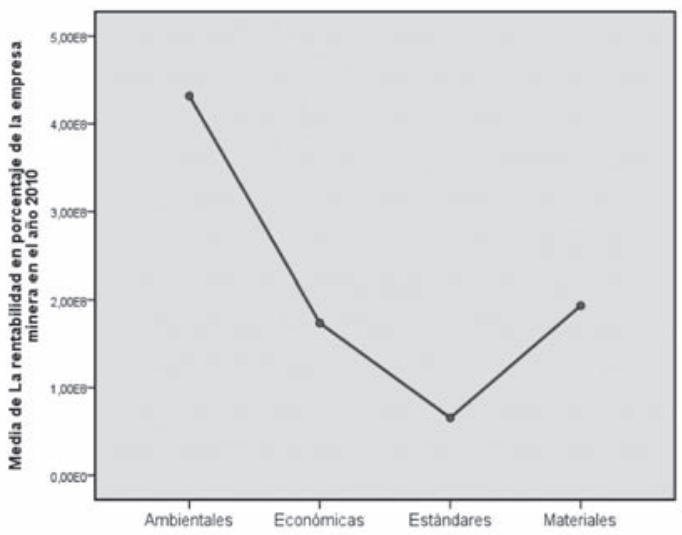

Fuente: Elaboración propia.

de 0.05 que indican un rechazo de la hipótesis H1. Los resultados obtenidos concuerdan con estudios realizados por otros autores, en los que no se presenta relación significativa entre la rentabilidad y el nivel de información sobre desempeño ambiental (Moneva y Llena, 1996; Archel y Lizarraga, 2001).

Tabla I: Prueba ANOVA - Rentabilidad.

Resumen de prueba de hipótesis

\begin{tabular}{|c|c|c|c|c|}
\hline & Hipótesis nula & Test & Sig. & Decisión \\
\hline 1 & $\begin{array}{l}\text { La distribución de La rentabilidad } \\
\text { en procentaje de la empresa minera } \\
\text { en el año } 2009 \text { es la misma entre } \\
\text { las categorias de Ward Method }\end{array}$ & $\begin{array}{l}\text { Prueba Kruskal- } \\
\text { Wallis de } \\
\text { muestras } \\
\text { independientes }\end{array}$ & 181 & $\begin{array}{l}\text { Retener la } \\
\text { hipótesis } \\
\text { nula. }\end{array}$ \\
\hline 2 & $\begin{array}{l}\text { La distribución de La rentabilidad en } \\
\text { porcentaje de la empresa minera en } \\
\text { el año } 2010 \text { es la misma entre las } \\
\text { categorias de Ward Method }\end{array}$ & $\begin{array}{l}\text { Prueba Kruskal- } \\
\text { Wallis de } \\
\text { muestras } \\
\text { independientes }\end{array}$ &, 075 & $\begin{array}{l}\text { Retener la } \\
\text { hipótesis } \\
\text { nula. }\end{array}$ \\
\hline
\end{tabular}

Se muestran las significancias asintóticas. El nivel de significancia es ,05

Fuente: Elaboración propia.

\section{Tamaño}

Con respecto a la variable tamaño, se ha analizado su relación con los componentes MR de desempeño con materiales y residuos, y con el componente EE, referido, principalmente, al consumo de energía y emisiones. Los Gráficos 4 y 5 muestran la media del nivel de información para estos componentes según el tamaño, donde las empresas de mayor tamaño presentan un mejor cumplimiento de estándares de información de la GRI.

La prueba de ANOVA (Tabla II) confirma que existe diferencia significativa en el nivel de cumplimiento de información en los componentes señalados según el tamaño. Con un p-valor de 0.001 menor al nivel de significancia de 0.05 , no se rechaza la hipótesis H2. Similares resultados se encuentran en estudios de Archel y Lizárraga (2001). 
Gráfico 4: Componente MR según tamaño.

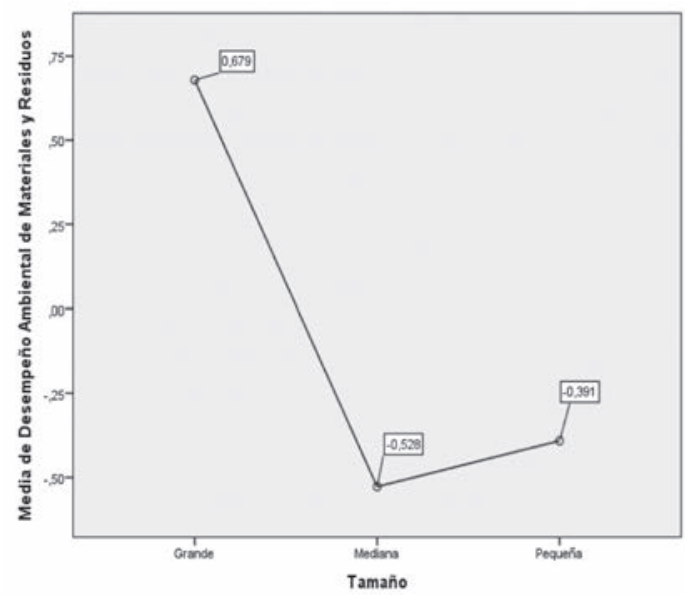

Fuente: Elaboración propia.
Gráfico 5: Componente EE según tamaño.

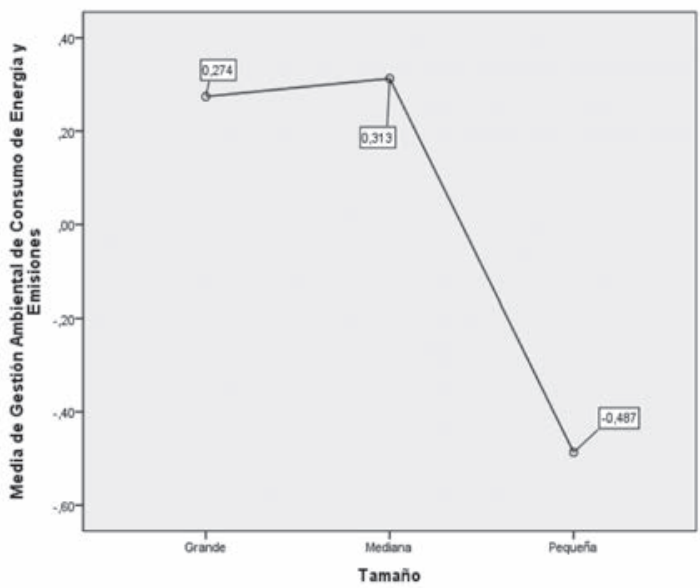

Fuente: Elaboración propia.

Tabla II: ANOVA para tamaño y componente MR.

\section{ANOVA de un factor}

Desempeño Ambiental de Materiales y Residuos

\begin{tabular}{|l|r|r|r|r|r|}
\hline & \multicolumn{1}{|c|}{$\begin{array}{c}\text { Suma de } \\
\text { cuadrados }\end{array}$} & gl & \multicolumn{1}{c|}{$\begin{array}{c}\text { Media } \\
\text { cuadrática }\end{array}$} & \multicolumn{1}{c|}{ F } & Sig. \\
\hline Inter-grupos & 13,065 & 2 & 6,532 & 8,561 &, 001 \\
Intra-grupos & 30,521 & 40 &, 763 & & \\
Total & 43,586 & 42 & & & \\
\hline
\end{tabular}

Fuente: Elaboración propia.

\section{Naturaleza de Accionistas}

Finalmente, para la variable naturaleza de los accionistas se ha considerado su relación con los componentes $\mathrm{AE}$ de desempeño ambiental económico y EE de consumo de energía y emisiones. Los Gráficos 6 y 7 muestran las medias del nivel de información de ambos componentes para la variable naturaleza de los accionistas, observándose que las empresas con capital extranjero presentan mejor cumplimiento de información que las de capital nacional. No obstante, las empresas de capital mixto presentan mejor nivel de cumplimiento en todos los casos. 
Gráfico 6: Componente AE según accionista.

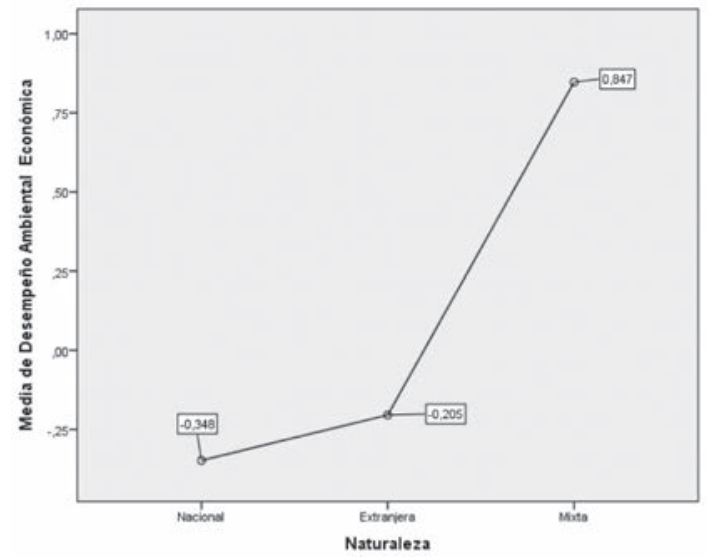

Fuente: Elaboración propia.

Se encontró diferencia significativa estadísticamente para el nivel de información del componente $\mathrm{AE}$ de desempeño ambiental económico. La Tabla III muestra la prueba de ANOVA
Gráfico 7: Componente EE según accionista.

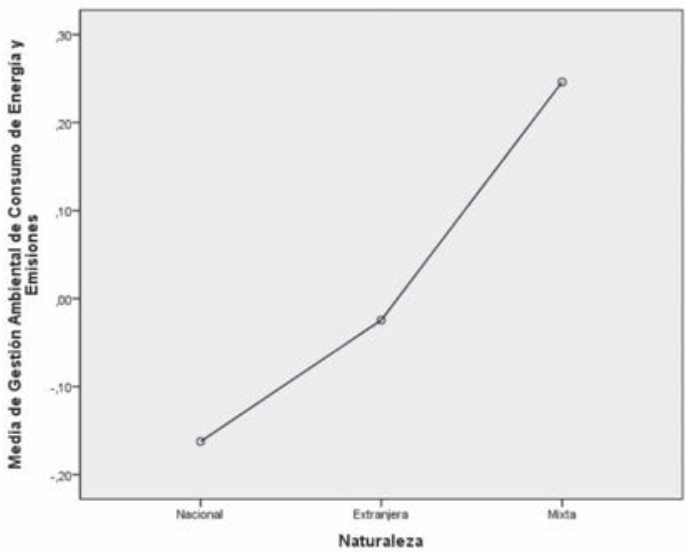

Fuente: Elaboración propia.

de no rechazo de la hipótesis $\mathrm{H} 3$ con un p-valor de 0.008 menor que el nivel de significancia de 0.05 .

Tabla III: ANOVA para naturaleza de los accionistas y componente AE.

ANOVA de un factor

Desempeño Ambiental Económica

\begin{tabular}{|l|r|r|r|r|r|}
\hline & \multicolumn{1}{|c|}{$\begin{array}{c}\text { Suma de } \\
\text { cuadrados }\end{array}$} & \multicolumn{1}{c|}{ gl } & \multicolumn{1}{c|}{$\begin{array}{c}\text { Media } \\
\text { cuadrática }\end{array}$} & \multicolumn{1}{c|}{ F } & \multicolumn{1}{c|}{ Sig. } \\
\hline Inter-grupos & 9,507 & 2 & 4,753 & 5,422 &, 008 \\
Intra-grupos & 35,068 & 40 &, 877 & & \\
Total & 44,575 & 42 & & & \\
\hline
\end{tabular}

Fuente: Elaboración propia.

Las grandes empresas con capitales extranjeros y locales presentan un mejor desempeño e información dado que poseen mayores recursos, estrategias corporativas de RSC definidas y claridad acerca de que el impacto ambiental es clave en la gestión con las comunidades campesinas como principal grupo de interés (Slack, 2012 y Pino et al., 2010).

\section{CONCLUSIONES}

En la actualidad la agenda sobre los impactos ambientales y sociales son determinantes en la estrategia de sostenibilidad y su información; para las empresas mineras peruanas el desempeño de sostenibilidad, la satisfacción de las expectativas de las comunidades campesinas, el cuidado de los recursos naturales y la gestión de residuos, resultan claves en el logro de una licencia social para la realización de sus operaciones.

Este desempeño es divulgado por las empresas mineras en sus memorias con distintos niveles de cumplimiento de información ambiental, según los criterios de la GRI, es así que el tamaño y naturaleza de los accionistas de las empresas mineras resultan factores determi- 
nantes del nivel de cumplimiento informativo; el cumplimiento resulta mayor para las empresas de mayor tamaño, recursos y participación de capitales extranjeros.

Se encuentra para las empresas mineras peruanas que el desempeño ambiental está dirigido principalmente a las prácticas de desempeño positivo relacionadas con el cuidado de los recursos naturales y tratamiento de residuos, la gestión con las comunidades en temas ambientales y sociales y el cumplimiento de la legislación ambiental, que pueden estar dirigidos a la búsqueda de una legitimidad social principalmente en las empresas de mayor tamaño y disposición de recursos (Gifford y Kestler, 2008).

Las grandes empresas mineras, que poseen mayores recursos y con importantes capitales extranjeros, realizan mejores inversiones y tienen más desarrolladas sus estrategias corporativas de sostenibilidad con las comunidades y sociedad, lo que puede explicar esta diferencia en el nivel de información desde un enfoque institucional.

Futuras investigaciones deben orientarse a un análisis de otros aspectos como la actitud de los gerentes, el enfoque estratégico, la influencia de las entidades públicas y la legislación como factores de divulgación ambiental.

\section{BIBLIOGRAFÍA}

Archel, D. y Lizarraga, P. (2001). Algunos determinantes de la información medioambiental divulgada por las empresas españolas cotizadas. Revista de Contabilidad, 4(7), 129-153.

Bebbington, A. y Bury, J. (2009). Institutional challenges for mining and sustainability in Peru. Proceedings of the National Academy of Sciences, 106(41), 17296-17301.

Da Silva, S. M., y Aibar, B. (2011). Factores determinantes del grado de información medioambiental divulgada en las grandes empresas que operan en Portugal: un análisis univariante. Base, 8(1).

Deegan, C. y Gordon, B. (1996). A study of the environmental disclosure practices of Australian corporations. Accounting and Business Research, 26(3), 187-199.

Fonseca, A., McAllister, M. y Fitzpatrick, P. (2014). Sustainability reporting among mining corporations: A constructive critique of the GRI approach. Journal of Cleaner Production, 70-83.

García-Ayuso, M. y Larrinaga, C. (2003). Environmental disclosure in Spain: Corporate characteristics and media exposure. Spanish Journal of Finance and Accounting, 115, pp. 184-214.

Gifford, B. y Kestler, A. (2008). Toward a theory of local legitimacy by MNEs in developing nations: Newmont mining and health sustainable development in Peru. Journal of International Management, 14, 340-352.

Gifford, B., Kestler, A. y Anand, S. (2010). Building local legitimacy into corporate social responsibility: Gold mining firms in developing nations. Journal of World Business, 45(3), 304-311.

Gray, R., Kouhy, R. y Lavers, S. (1995). Corporate social and environmental reporting: A review of the literature and a longitudinal study of UK disclosure. Accounting, Auditing and Accountability Journal, 8(2), 47-77.

Gray, R., Owen, D. y Adams, C. (1996). Accounting and accountability: Changes y challenges in corporate social and environmental reporting. London: Prentice Hall.

GRI (Global Reporting Initiative) (2006). Sustainability reporting guidelines. Amsterdam.

GRI (Global Reporting Initiative) (2010). Sustainability reporting guidelines, mining, and metals sector supplement. Amsterdam.

Guenther, E., Hoppe, H. y Poser, C. (2006). Environmental corporate social responsibility of firms in the mining and oil and gas industries. Greener Management International, Vol. 53, 7-25.

Helwege, A. (2014). Challenges with resolving mining conflicts in Latin America. The Extractive Industries and Society, 2(1), 73-84.

Hilson, G. y Basu, A. (2003). Devising indicators of sustainable development for the mining and minerals industry: An analysis of critical background issues. The International Journal of Sustainable Development and World Ecology, 10(4), 319-332.

Jaskoski (2014). Environmental licensing and conflict in Peru's mining sector: A path-depen- 
dent analysis. World Development, (64), 873-883.

Jenkins, H. (2004). Corporate social responsibility and the mining industry: Conflicts and constructs. Corporate Social Responsibility and Environmental Management, 11(1), 23-34.

Jenkins, H. y Yakovleva, N. (2006). Corporate social responsibility in the mining industry: Exploring trends in social and environmental disclosure. Journal of Cleaner Production, 14(3-4), 271-284.

Llena, F., Moneva, J. M. y Hernández, B. (2007). Environmental disclosures and compulsory accounting standards: The case of Spanish annual reports. Business Strategy and the Environment, 16(1), 16-53.

Moneva, J. M. y Llena, F. (1996). Análisis de la información sobre responsabilidad social en las empresas industriales que cotizan en bolsa. Revista Española de Financiación y Contabilidad, 25(87), 361-401.

Moneva, J. M., Acero, I. y Llena, F. (2007). Evaluación de la información de sostenibilidad en las cajas de ahorro españolas. Cuadernos Aragoneses de Economía, 17(1), 99-125.

Muradian, R., Martínez-Alier, J. y Correa, H. (2003). International capital vs. local population: The environmental conflict of the Tambogrande mining project. Society and Natural Resources, 16(9), 775-792.

Patten, D. M. (1992). Intra-industry environmental disclosures in response to the Alaskan oil spill: A note on Legitimacy Theory. Accounting, Organizations and Society, Vol. XVII, $\mathrm{N}^{\circ}$, 471-475.

Patten, D. M. (2002). The relation between environmental performance and environmental disclosure: A research note. Accounting, Organizations and Society, 27(8), 763-773.

Pérez, F. y Sánchez, L. (2009). Assessing the evolution of sustainability reporting in the mining sector. Environmental Management, 43, 949-961.

Pino, R., Aquije, E., Cárdenas, A. y Moreno, P. (2010). La actividad minera y su relación con las comunidades: un estudio exploratorio en el Perú. CENTRUM, PUCP. Lima.
Prno, J. (2013). An analysis of factors leading to the establishment of a social licence to operate in the mining industry. Resources Policy, 38(4), 577-590.

Roberts, R. (1992). Determinants of corporate social responsibility disclosure: An application of stakeholder theory. Accounting, Organizations and Society, 17(6), 595-612.

Slack, K. (2012). Mission impossible?: Adopting a CSR-based business model for extractive industries in developing countries. Resources Policy, 37(2), 179-184.

Sweeney, L. y Coughlan, J. (2008). Do different industries report corporate social responsibility differently? An investigation through the lens of stakeholder theory. Journal of Marketing Communications, 14(2), 113-124. 
